\title{
Laser-Induced Nuclear Processes in Ultra-Dense Hydrogen Take Place in Small Non-superfluid $\mathrm{H}_{N}(0)$ Clusters
}

\author{
Leif Holmlid $^{1}$
}

Received: 31 May 2018/Published online: 3 December 2018

(C) The Author(s) 2018

\begin{abstract}
Charged and neutral kaons are formed by impact of pulsed lasers on ultra-dense hydrogen $\mathrm{H}(0)$. This superfluid material $\mathrm{H}(0)$ consists of clusters of various forms, mainly of the chain-cluster type $\mathrm{H}_{2 N}$. Such clusters are not stable above the transition temperature from superfluid to normal matter. In the case studied here, this transition is at $525 \mathrm{~K}$ for $\mathrm{D}(0)$ on an $\mathrm{Ir}$ target, as reported previously. Mesons are formed both below and above this temperature. Thus, the meson formation is not related to the long chain-clusters $\mathrm{H}_{2 N}$ but to the small non-superfluid cluster types $\mathrm{H}_{3}(0)$ and $\mathrm{H}_{4}(0)$ which still exist on the target above the transition temperature. The nuclear processes forming the kaons take place in such clusters when they are transferred to the lowest $s=1$ state with $\mathrm{H}-\mathrm{H}$ distance of $0.56 \mathrm{pm}$. At this short distance, nuclear processes are expected within $1 \mathrm{~ns}$. The superfluid chain-cluster phase probably has no direct importance for the nuclear processes. The clusters where the nuclear processes in $\mathrm{H}(0)$ take place are thus quite accurately identified.
\end{abstract}

Keywords Ultra-dense hydrogen $\cdot$ Superfluid $\cdot$ Picometer $\cdot$ Transition temperature

\section{Introduction}

The nuclear processes taking place in ultra-dense hydrogen $\mathrm{H}(0)$ under pulsed-laser impact give mesons showers [1]. These showers are similar to those from nucleon-antinucleon annihilation processes [2]. Nuclear processes in $\mathrm{H}(0)$ are possible due to the short distance between the nuclei in the ultra-dense form: at the most common spin level $s=2$ [3-5] the $\mathrm{H}-\mathrm{H}$ distance is $2.245 \pm 0.003 \mathrm{pm}$ (recalculated from accurate data with $s=3$ ) [6]. At the lowest spin level $s=1$, the distance is only $0.56 \mathrm{pm}$ [3]. This distance is expected to give nuclear reactions within $1 \mathrm{~ns}$, as known from studies of muon catalyzed fusion [7] where the internuclear distance is $0.51 \mathrm{pm}(106 / 207 \mathrm{pm})$. The details of the nuclear process in $\mathrm{H}(0)$ need to be studied, so that the process can be optimized: it gives the possibility of energy production with a previously unknown efficiency, hundred times better than ordinary fusion. Here, the $\mathrm{H}(0)$

Leif Holmlid

holmlid@chem.gu.se

1 Atmospheric Science, Department of Chemistry and Molecular Biology, University of Gothenburg, SE-412 96 Göteborg, Sweden clusters in which the nuclear processes take place are identified. It is concluded that the long superfluid chaintype clusters $\mathrm{H}_{2 N}$ are not directly involved. Instead, small non-superfluid clusters like $\mathrm{H}_{3}(0)$ and $\mathrm{H}_{4}(0)$ [8] which have no specific axis give the nuclear processes after transfer to the lowest spin state with spin $s=1$ [3].

\section{Theoretical Background}

An ultra-dense form of Rydberg matter [3-5, 9, 10] has been studied during a few years time. These studies have identified two slightly different forms, ultra-dense protium $\mathrm{p}(0)$ [9] and ultra-dense deuterium $\mathrm{D}(0)[3,10]$. In ordinary Rydberg matter (RM) [5], the electron orbital angular momentum $l$ is always $>0$. In the ultra-dense material which can only be formed from hydrogen, $l=0$; its structure is instead given by the spin angular momentum $s>0$. This quantum number was identified from laser-induced time-of-flight (TOF) and time-of-flight mass spectrometry (TOF-MS) studies [4, 10-12] to have values $s=1,2$ or 3 , giving an interatomic distance of only $0.56 \mathrm{pm}$ in level $s=1$ [3]. Recently, also optical spectroscopy was used to give much more precise bond 
distances for levels $s=2,3$ and $4[6,13]$. The density of the ultra-dense material is around $10^{29} \mathrm{~cm}^{-3}$, corresponding to a theoretical D-D distance at $s=2$ of $2.23 \mathrm{pm}$ [3] and the normal experimental distance in the TOF-MS studies of $2.3 \pm 0.1 \mathrm{pm}[4,10-12]$. Most experiments with ultra-dense hydrogen have in fact studied ultra-dense deuterium $\mathrm{D}(0)$ due to its slightly simpler structure, with less interaction between the nuclei due to their Boson properties. The quantum mechanical basis for $\mathrm{D}(0)$ was first discussed by Winterberg [14, 15], pointing out the similarity to other superfluids [16]. Theory [17-19] predicts a dense deuterium phase with both superfluid and superconducting properties.

Experimental studies of clusters of ultra-dense hydrogen $\mathrm{H}(0)$ show that they are chain clusters of the form $\mathrm{H}_{2 N}$ with $N$ an integer. This form is shown at the top in Fig. 1. Each $\mathrm{H}_{2}$ "bead" is formed by a pair $\mathrm{H}-\mathrm{H}$ which rotates around the cluster axis. Each such cluster shows a Meissner effect, thus it floats in a static magnetic field $[8,20]$. This is characteristic for a superconducting material. Hirsch [21] describes the superconducting state of a material as having large Rydberg-like orbits. This is similar to an ordinary RM cluster state with $l>0$, but here with the plane of the orbit given by the magnetic field direction, not by the geometry of the cluster as in the case of an ordinary (orbital angular momentum-based) RM cluster. Only one or a few of the electrons in each cluster are simultaneously in such high Rydberg-like states. These electrons are the ones most easily displaced by the laser-pulse field.

Both forms of $\mathrm{H}(0)$, ultra-dense protium $\mathrm{p}(0)$ [9] and ultra-dense deuterium $\mathrm{D}(0)[3,10]$, are superfluid at room temperature [22] as shown for example by a fountain effect

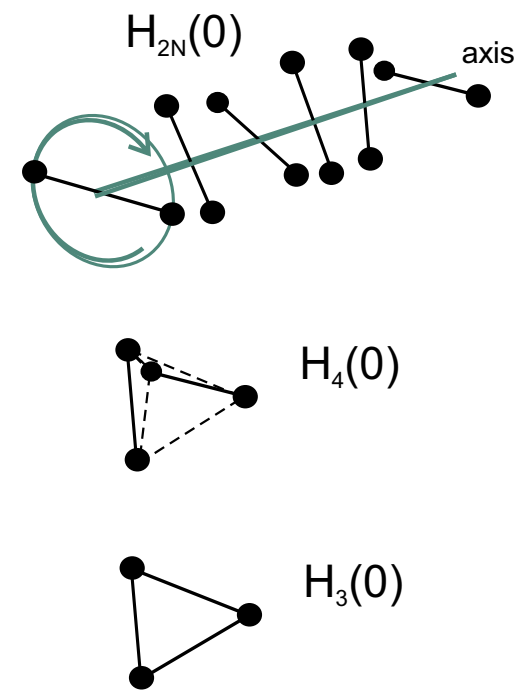

Fig. 1 Shape of the chain or "bead" clusters $\mathrm{H}_{2 N}(0)$ forming the superfluid phase $\mathrm{H}(0)$ and non-superfluid cluster types $\mathrm{H}_{3}(0)$ and $\mathrm{H}_{4}(0)$ and by the fast laser energy transport. They also show a Meissner effect at room temperature, which means that the long chain-like clusters lift in a static magnetic field $[8,20]$. This effect is less pronounced for $\mathrm{p}(0)$ [20], presumably due to its slightly more complex cluster structure compared to $\mathrm{D}(0)[8,9]$. This difference is probably due to the formation of proton pairs which gives three protons $p_{3}$ coupled together instead of just a proton pair $\mathrm{p}_{2}$ in a "bead" [9]. Due to the large difference in scale between the ultra-dense material and the metal carrier surface (typically $2 \mathrm{pm}$ instead of $200 \mathrm{pm}$ for the carrier), many novel effects are expected. It means for example that an entire chain cluster $\mathrm{H}_{2 N}$ may fit in between two metal atoms on the surface.

In the Meissner experiments using $\mathrm{D}(0)$ [8], it was clearly observed that small clusters $\mathrm{D}_{3}(0)$ and $\mathrm{D}_{4}(0)$ do not float in the magnetic field, thus they do not show a Meissner effect. Long chain clusters $\mathrm{D}_{2 N}$ on the other hand float in the static magnetic field. The small clusters do not have a long axis due to their symmetry as shown at the bottom in Fig. 1. It was concluded in Ref. [22] that such small clusters probably do not form a superfluid layer on the metal carrier surface used in the experiments. These results indicate that a material formed from such small symmetric ultra-dense clusters will not have superfluid or superconductive properties. In the TOF studies, these small clusters give a signal clearly different from the long chainclusters. This is here used to identify the clusters in which the nuclear processes take place.

The decay signal is measured at two collectors (or one coil) in the beam ejected from the pulsed-laser interaction with ultra-dense hydrogen $\mathrm{H}(0)$. The laser pulse has a typical width of $7 \mathrm{~ns}$, with a rise-time of $3 \mathrm{~ns}$. The signal at the collectors (and at the coil) is in the form of a pulse with quite well-defined rise and fall times. This means that the signal is due to an intermediate particle $\mathrm{M}$ which is formed and decays like $\mathrm{A} \rightarrow \mathrm{M} \rightarrow \mathrm{N}$ [23]. Often, approximately the same time dependence is observed at both collectors, which means that slow decaying particles $\mathrm{M}$ at the target give the fast detected particles $\mathrm{N}$ at the collectors.

The time dependence of the signal $\mathrm{M}$ is easily derived from the rate equations for the process $\mathrm{A} \stackrel{k_{1}}{\longrightarrow} \mathrm{M} \stackrel{k_{2}}{\longrightarrow} \mathrm{N}$ [23]

$-\frac{d n_{\mathrm{A}}}{d t}=k_{1} n_{\mathrm{A}}$

$\frac{d n_{\mathrm{M}}}{d t}=k_{1} n_{\mathrm{A}}-k_{2} n_{\mathrm{M}}$

as

$n_{\mathrm{M}}=\frac{k_{1}}{k_{2}-k_{1}} n_{\mathrm{A} 0}\left(e^{-k_{1} t}-e^{-k_{2} t}\right)$

where $n_{\mathrm{A} 0}$ is the number density of the precursor at time $t=0$ thus at the start of the laser pulse. This derivation puts 
the initial number density of the intermediate meson $n_{\mathrm{M} 0}$ to zero. The curve shape in Eq. (3) is used to match the results below. The results are given as time constants $\tau=1 / k$.

\section{Experimental}

The apparatus is shown in Fig. 2. It has been described in several publications, for example in Ref. [10]. It has a base pressure of $<1 \times 10^{-6}$ mbar. The central source part is described in Ref. [4]. The emitter is a cylindrical (extruded) sample of an industrial iron oxide catalyst doped with $\mathrm{K}$ $[24,25]$, a so called styrene catalyst type Shell S-105 (obsolete type number). This type of catalyst is an efficient hydrogen abstraction and transfer catalyst. The source metal tube can be heated by an AC current through its wall up to $400 \mathrm{~K}$. Deuterium gas $(99.8 \%)$ is admitted through the tube at a pressure up to $1 \times 10^{-5}$ mbar in the chamber.

The metal target is constructed for direct heating with a $50 \mathrm{~Hz}$ AC current. The carrier $\mathrm{Ni}$ foil with dimension $12 \times 15 \mathrm{~mm}$ has a thickness of $0.2 \mathrm{~mm}$. It is spot welded to two thinner foils of Ta with thickness $0.1 \mathrm{~mm}$ which carry the heating current. On the carrier, short Ir rods with $2 \mathrm{~mm}$ diameter are spot-welded. The carrier foil is mounted at $45^{\circ}$ to the vertical direction and located approximately $1 \mathrm{~cm}$ below the source tip. The heating current through the carrier and its supporting foils is taken from an external ring transformer with a few turns of secondary winding. The temperature of the carrier is measured by a type $\mathrm{K}$ thermocouple (TC), spot welded at the upper half of the carrier foil. The cold end of the TC is at the screw support on the arms holding the carrier foil, at a distance of $20 \mathrm{~cm}$ from the carrier.

A Nd:YAG laser with an energy of $<125 \mathrm{~mJ}$ in $5 \mathrm{~ns}$ long pulses at $10 \mathrm{~Hz}$ is used at $532 \mathrm{~nm}$. The laser beam is focused on the carrier at the center of the chamber with an $f=400 \mathrm{~mm}$ spherical lens. The lens is mounted in a vertical motion translation stage. The intensity in the beam waist of (nominally) $70 \mu \mathrm{m}$ diameter is relatively low, $\leq 10^{12} \mathrm{~W} \mathrm{~cm}^{-2}$ as calculated for a Gaussian beam. A dynode-scintillator-photomultiplier detector which

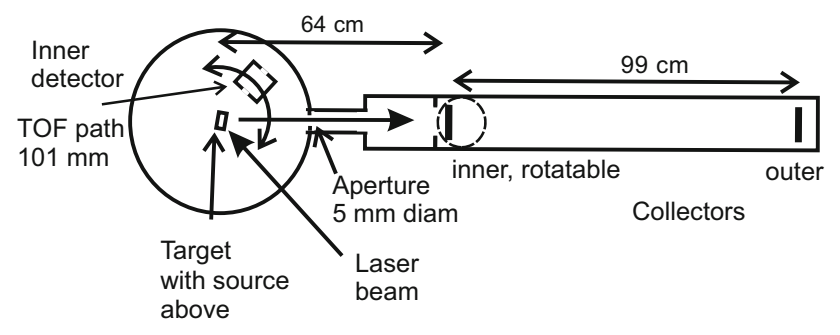

Fig. 2 Horizontal cut through the apparatus measures the time-of-flight (TOF) spectra of the neutral and ionized flux from the laser initiated processes is shown in Fig. 2. This detector can be rotated around the carrier at the center of the chamber. Fast neutral particles impact on a steel catcher foil in the detector, and ions ejected from there (or in the beam) are drawn towards a $\mathrm{Cu}-\mathrm{Be}$ dynode held at $-7.0 \mathrm{kV}$ inside the detector. The total effective flight distance for the particles from the laser focus to the catcher foil is $101 \mathrm{~mm}$ by direct measurement and internal calibration $[5,10]$. The photomultiplier (PMT) is Electron Tubes 9128B (single electron rise time of $2.5 \mathrm{~ns}$ and transit time of $30 \mathrm{~ns}$ ). This PMT is covered by Al foil and black plastic tape giving only a small active cathode area of 2-3 $\mathrm{mm}^{2}$ to avoid saturation. Blue glass filters in front of the PMTs remove any scattered laser light. A fast preamplifier (Ortec VT120A, gain 200, bandwidth 10-350 MHz) is used. The signal from the PMT is sometimes collected on a fast digital oscilloscope (Tektronix TDS 3032, $300 \mathrm{MHz}$ ). The average function in the oscilloscope is used. A multi-channel scaler (MCS) with 5 ns dwell time per channel is used (EG\&G Ortec Turbo-MCS) for the TOF-MS spectra. Each MCS spectrum consists of the sum of 500 laser shots.

The laser-induced mass spectrometry used here is described in several publications [3, 4, 10-12]. Due to the very short bond distances in ultra-dense hydrogen and also in low levels of ordinary hydrogen Rydberg matter $H(1)$ and $H(2)$, the kinetic energy release (KER) given to the cluster fragments by the Coulomb explosions (CE) is quite high. It is also well-defined, due to the easy removal of the orbiting Rydberg electrons by the laser pulse, without any large changes of the structure before the fs long repulsion period between the fragments [26]. The total kinetic energy of the fragments gives directly their initial distance as

$r=\frac{1}{4 \pi \varepsilon_{0}} \frac{e^{2}}{E_{k i n}}$

where $\varepsilon_{0}$ is the vacuum permittivity, $e$ the unit charge on the fragment ions and $E_{k i n}$ the sum kinetic energy for the fragments (KER) from the CE. The fraction of the KER that is observed on each fragment depends on the mass ratio of the fragments. The kinetic energy is determined by measuring the time-of-flight (TOF) of the fragments and then converting this quantity to kinetic energy. In the case of long $\mathrm{H}(0)$ chain clusters, a central fragmentation is often observed, thus giving two identical cluster fragments, each carrying half the total KER. The most common state of $\mathrm{H}(0)$ has $s=2$ [3]. It has $2.3 \mathrm{pm} \mathrm{H}-\mathrm{H}$ distance and gives a total KER of $640 \mathrm{eV}$.

Part of the flux from the laser-induced processes on the target is taken out through a small opening in the chamber wall to a separately pumped and valved chamber with two 
collectors consisting of a metal wire loop covered with 1-2 layers of $20 \mu \mathrm{m}$ thick $\mathrm{Al}$ foil. The loops have a diameter of $50 \mathrm{~mm}$ inside the tube with internal diameter $63 \mathrm{~mm}$. The collectors are at a distance of $66 \mathrm{~cm}$ (inner collector) and $163 \mathrm{~cm}$ (outer collector) from the target as seen in Fig. 2. The signal to them is directly observed on a fast digital oscilloscope (Tektronix TDS 3032, $300 \mathrm{MHz}$ ). The oscilloscope is triggered by a pulse from a photodiode close to the laser. The diode is located such that the trigger delay in the cable to the oscilloscope is close to the time for the pulse to move to the target and through the cable to the oscilloscope. The error is estimated to be $\pm 1-2$ ns [27]. The typical behavior of the signals is shown in several publications $[1,23]$. The decay times of these signals are characteristic for the decaying mesons. The signal is normally due to fast (relativistic) muons arriving to the collectors from the relatively slow decaying mesons (kaons and pions).

In some tests the inner collector is replaced by a wire coil wound on a ferrite toroid. This coil works as a current transformer for the particle beam current. The signal is due to charged fast particles, and photons and other neutral particles like neutral kaons cannot be observed by this coil. A direct comparison of the coil signal and the outer collector signal shows that the same particles are observed, thus proving that the outer collector signal is due to fast charged particles and not to photons or neutral particles. The measuring coil is wound on a N30 MnZn soft ferrite toroid with epoxy cover (EPCOS) with inner diameter $25 \mathrm{~mm}$. There are 19 turns of enameled copper wire wound on it in the negative direction. The toroid hangs freely in the conductor wires in the particle beam. One end of the coil is in turn connected to the $50 \Omega$ oscilloscope input, while the other end is connected to a $50 \Omega$ BNC termination to the mounting flange. The sign of the beam current is determined by calibration using a current pulse in a single wire through the opening of the coil, to avoid possible sign errors.

\section{Results}

The temperature variation of the $\mathrm{H}(0)$ clusters on the foil target was studied with the inner detector shown in Fig. 2 in Ref. [28]. It was demonstrated that the long chainclusters $\mathrm{H}_{2 N}(0)$ disappear at a transition temperature in the range 405-725 K, varying with the carrier material and the ultra-dense hydrogen form, being protium $\mathrm{p}(0)$ or deuterium $\mathrm{D}(0)$. Results for Ir as the surface and with $\mathrm{D}(0)$ as the ultra-dense hydrogen are shown in Fig. 3. The shortest TOF possible for different excitation levels in deuterium is shown in Table 1. These data are based on extensive studies $[3,10-12,22]$. The large chain-clusters $\mathrm{D}(0)$ are

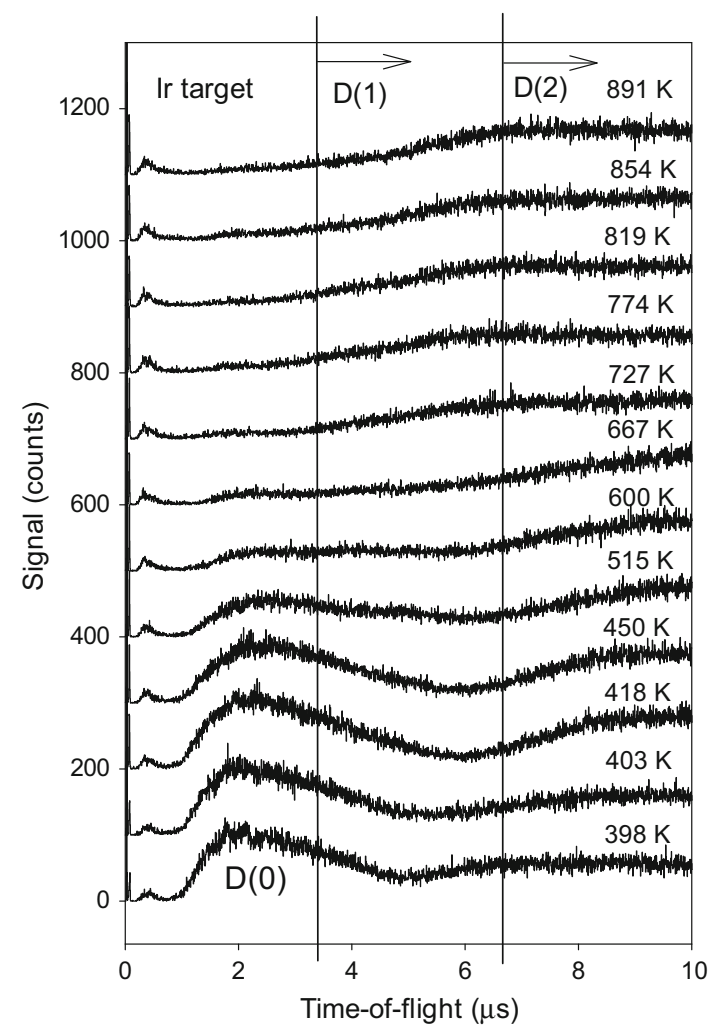

Fig. 3 TOF spectra at $45^{\circ}$ detector position relative to laser beam. $\mathrm{D}(0)$ on Ir surface. Temperature variation shown

Table 1 The shortest possible TOF for D (repelled from infinite mass) from symmetric and asymmetric $\mathrm{CE}$ processes with 2 and 3 charges in the $\mathrm{D}(\mathrm{RM})$ clusters [12]

\begin{tabular}{lcll}
\hline Excitation level & KER $(\mathrm{eV})$ & CE 2+ & CE 3+ \\
\hline $\mathrm{D}(l=0, s=1)$ & 2586 & $204 \mathrm{~ns}$ & \\
$\mathrm{D}(l=0, s=2)$ & 647 & $408 \mathrm{~ns}$ & \\
$\mathrm{D}(l=0, s=3)$ & 287 & $612 \mathrm{~ns}$ & \\
$\mathrm{D}(1)$ & & $3.4 \mu \mathrm{s}$ & $2.4 \mu \mathrm{s}$ \\
$\mathrm{D}(2)$ & & $6.7 \mu \mathrm{s}$ & \\
$\mathrm{D}(3)$ & & $10.1 \mu \mathrm{s}$ & \\
$\mathrm{D}(4)$ & & $13.5 \mu \mathrm{s}$ & \\
\hline
\end{tabular}

These times are indicated by vertical lines in the TOF spectra

found in the time range $1-4 \mu \mathrm{s}$, only at temperatures below $525 \mathrm{~K}$ [28]. They give relatively large fragments, and not atoms $\mathrm{D}$ or atomic ions $\mathrm{D}^{+}$. The fastest part of the signal is $\mathrm{D}$ atoms from small $\mathrm{D}(0)$ clusters at $200-500 \mathrm{~ns}$ TOF. A sum of all distributions from all temperatures is shown in Fig. 4. A large part of this distribution comes from $D(0)$ in the lowest level with $s=1$, at a D-D distance of $0.56 \mathrm{pm}$. At such short distances, nuclear processes are expected to be fast [7]. A large peak at $20-40 \mathrm{~ns}$ in Fig. 4 also exists, which has not been assigned previously. It may be due to 


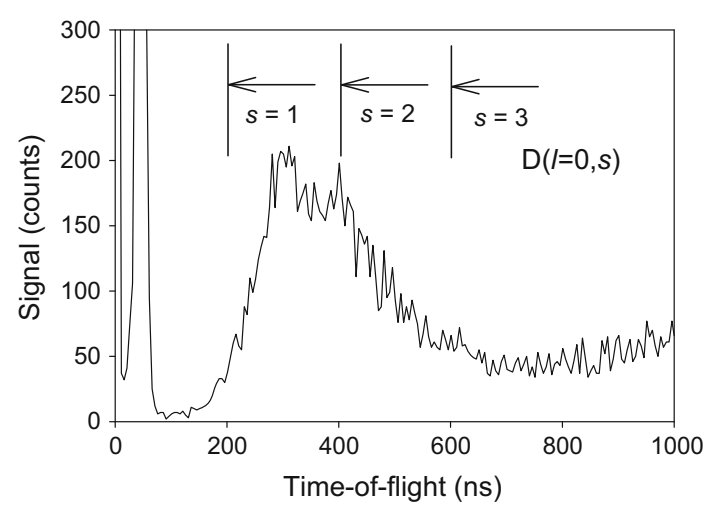

Fig. 4 Sum of all the TOF spectra in Fig. 3, showing that the distribution peaking at 300-400 ns contains a large contribution from the lowest level $s=1$ in which nuclear processes are expected

processes giving a typical nuclear based energy of $100 \mathrm{keV} \mathrm{u}^{-1}$.

The MeV TOF signal to the inner and outer collectors shown in Fig. 2 was studied in Ref. [1]. The collector signals have the time dependence of an intermediate particle in a decay chain [23], with the decay times equal to the meson lifetimes $12.4 \mathrm{~ns}$ for charged kaons $\mathrm{K}^{ \pm}, 26 \mathrm{~ns}$ for charged pions $\pi^{ \pm}$and $52 \mathrm{~ns}$ for long-lived neutral kaons $\mathrm{K}_{L}^{0}$. In Fig. 5, the signal at the outer collector is shown to agree with the decay time for charged kaons, with the Ir target both cold and warm (at approximately $800 \mathrm{~K}$ ). This temperature is considerably higher than the transition temperature for $\mathrm{D}(0)$ on Ir, which is $525 \mathrm{~K}$ [28]. Thus, the
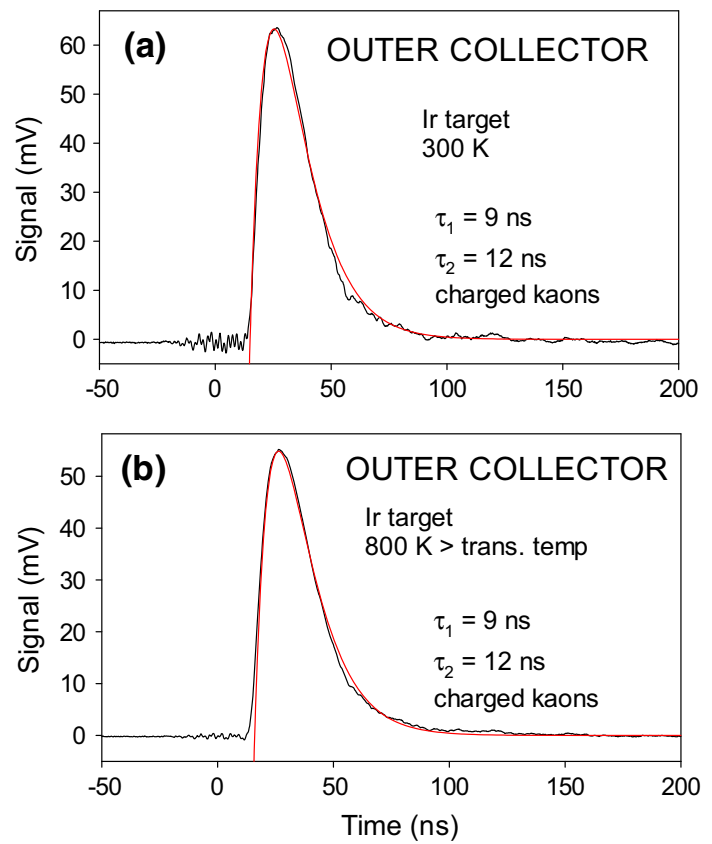

Fig. 5 Charged kaon signal at the outer collector in Fig. 2 with bias $-24 \mathrm{~V}$. The meson signal exists even at a temperature of $800 \mathrm{~K}$, above the $\mathrm{D}(0)$ on Ir transition temperature of $525 \mathrm{~K}$ [28] meson signal is formed also above the transition temperature where the chain-clusters are no longer stable. In the same experiment, the results in Fig. 6 were found for the inner collector. This signal is more complex and shows contributions from both charged pions and charged kaons. The signals at high and low temperature were identical, and thus only the high temperature signal is shown here. Since this signal is measured at relatively short distance from the target, it may also contain contributions from no yet decayed pions, not only from the muons formed by pion decay. This may give the slightly worse fit of the calculated curves in this case relative to Fig. 5 for example.

That the signals at the inner and outer collectors contain different contributions from mesons is often observed, and it is difficult to control the various meson contributions at the nuclear level to the meson shower in the experiments. This effect may sometimes be due to the different energies and angular widths of the signal distributions finally decaying to the muons observed as a signal current to the collectors. The initially formed kaons (neutral and charged) have quite low kinetic energy while the pions which are mainly formed from decaying neutral kaons [29-31] have much higher transverse translational energy. This means that some of them move out from the beam: their muon decay products may thus not reach the outer collector with the same probability. However, the main charged kaon decay channel gives directly fast muons [29-31] which may be more easily observed at the outer collector. The important point is that mesons are formed even at high target temperature: thus they are formed from the small $\mathrm{D}(0)$ clusters that are the only $\mathrm{D}(0)$ clusters which exist on the target at high temperature.

The only uncertainty in the collector measurements is the exact (at least the dominant) sign of charge of the observed particles. It may also be important to exclude that

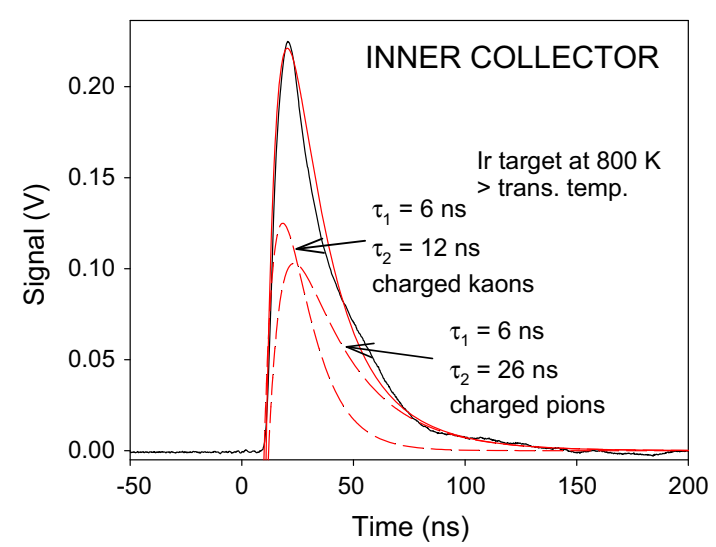

Fig. 6 Combined charged kaon and pion signal at the inner collector in Fig. 2 with bias $-24 \mathrm{~V}$. The meson signal exists even at a temperature of $800 \mathrm{~K}$, above the $\mathrm{D}(0)$ on Ir transition temperature of $525 \mathrm{~K}[28]$ 
this signal is due to photons. These problems have been solved by replacing the inner collector with a coil for a positive (direct) observation of the particle current in the beam. The difference between the signals at the two ends of the coil is the particle current signal. Results are shown in Figs. 7 and 8. In these figures (coil and outer collector measured in the same experiment) the signal in this case is due to the sum of charged kaons and charged pions decaying to muons, in a chain $\mathrm{K}^{ \pm} \rightarrow \pi^{ \pm} \rightarrow \mu^{ \pm}$[29-31]. (Note that the pions $\pi^{ \pm}$are allowed to have a rise time of $12 \mathrm{~ns}$ equal to the decay time of the kaons $\mathrm{K}^{ \pm}$as required for this decay chain). That the signal due to pions is lower in the coil than at the collector may be due to the lower kinetic energy of the muons from pion decay (maximum $139 \mathrm{MeV}$ ) relative to the muons from the direct kaon decay (not via pions, maximum $493 \mathrm{MeV}$ ). Since the amplitude of the signal in the coil varies with the velocity of the charges passing through, the kaon generated muons may give a larger induced signal per charge. The important points are that charged particles (pions and kaons) are indeed observed at the collector and that the collector signal is not due to photons. The sign of the current is verified to be positive from separate tests with a wire through the coil. The agreement between the meson decay results at high and low target temperature and the coil measurements is reassuring.

\section{Discussion}

The laser experiments sample the surface layer of $\mathrm{D}(0)$ on the target. Two different types of processes are initiated by the laser pulse. The first type of process is Coulomb

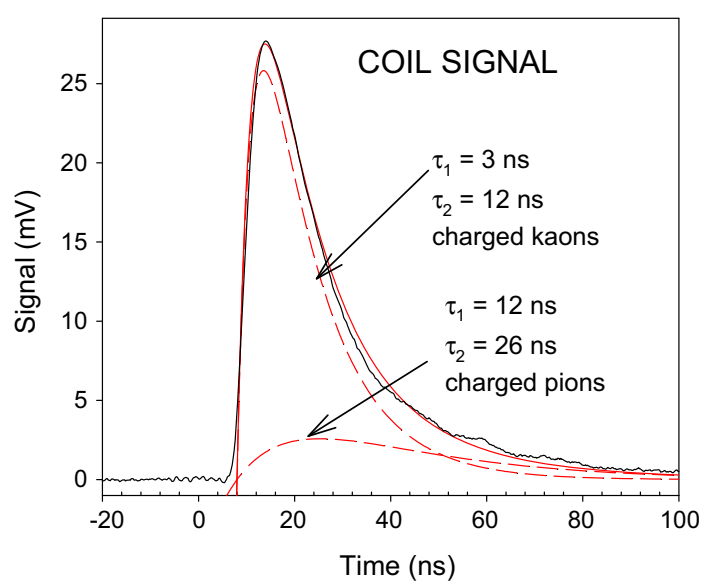

Fig. 7 Current coil signal with $\mathrm{D}(0)$ on target. Coil at position of inner collector in Fig. 2. Note that the pions $\pi^{ \pm}$have rise time of $12 \mathrm{~ns}$ equal to the decay time of the kaons $\mathrm{K}^{ \pm}$as required for the decay chain $\mathrm{K}^{ \pm} \rightarrow \pi^{ \pm} \rightarrow \mu^{ \pm}$. The actual particle current is probably due to muons

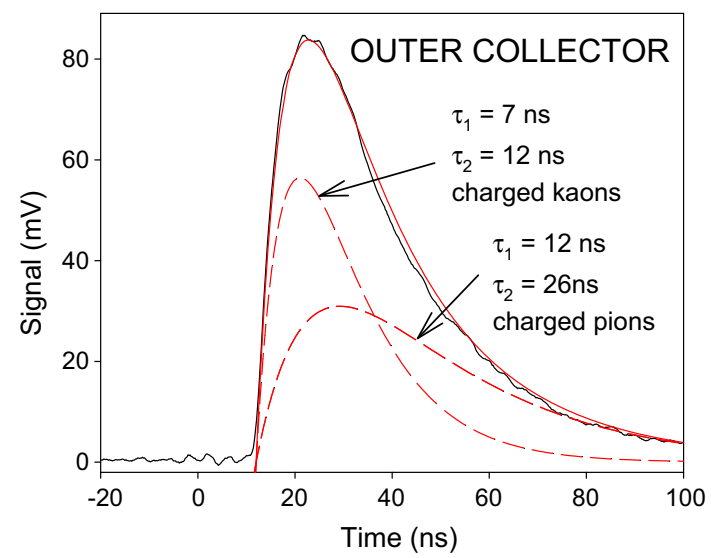

Fig. 8 Signal at outer collector with bias $-24 \mathrm{~V}$ with the coil in the beam in the same experiment as in Fig. 7. Note that the pions $\pi^{ \pm}$have rise time of $12 \mathrm{~ns}$ equal to the decay time of the kaons $\mathrm{K}^{ \pm}$as required for the decay chain $\mathrm{K}^{ \pm} \rightarrow \pi^{ \pm} \rightarrow \mu^{ \pm}$. The actual signal is probably due to muons giving secondary electron ejection at collector

explosions $(\mathrm{CE})$ in the $\mathrm{D}(0)$ clusters $[4,5,10]$ giving ions and atoms at maximum kinetic energy of $2.6 \mathrm{keV}$ as shown in Figs. 3 and 4 and Table 1. This process is initiated by the laser photons which remove electrons from the clusters (mainly the highly excited superconductive electrons in Rydberg-like orbits [21]). The second type of process is nuclear giving meson ejection followed by meson decay $[1,27,32,33]$, with final particle kinetic energies in the range up to $500 \mathrm{MeV}$. This process is instead initiated by the transfer of the clusters from spin level $s=2$ to $s=1$ which is the lowest level in $\mathrm{D}(0)$ having an internuclear distance of only $0.56 \mathrm{pm}$ [10]. It is possible that these two processes exist at different locations relative to the center of the laser beam, with the nuclear processes closer to the beam center and the $\mathrm{CE}$ processes in the outskirts of the laser beam. In general, the best CE experiments (TOF and TOF-MS) are done at lower laser intensity than the best meson experiments, so they are normally not performed under identical laser intensity conditions. The difference in laser intensity is however less than a factor of two, which means that the changes in the surface layer between these two experimental types are rather small. Thus, the results from these two types of experiment can be compared to each other.

The observed short interatomic $\mathrm{H}-\mathrm{H}$ distances in the pm range $[4,5,10]$ are also related to magic numbers and super heavy elements as found by Prelas et al. [34]. The idea is that deuterons at the common $2 \mathrm{pm}$ interatomic distance (which corresponds to $s=2$ ) may build Bose-Einstein clusters [34].

The typical total particle number ejected in the meson experiments is $10^{12}-10^{13}$ per laser pulse [1, 35], with energy (velocity) of $10-500 \mathrm{MeV} \mathrm{u}^{-1}$. The total number of particles ejected in the CE experiments is more difficult to 
estimate due to the much more complex signal conversion chain needed for the ejected low energy ions and atoms, in the typical initial energy range of only $320-640 \mathrm{eV}$. This number is here estimated to be in the range of $10^{6}-10^{9}$ per laser pulse. Numerous particles are formed with lower energy than $300 \mathrm{eV}$, but they are not included here.

The conclusion that the mesons originate from the small $\mathrm{H}(0)$ clusters is also based on the assumption that no other type of $\mathrm{D}(0)$ is still stable at high temperature and can be excited by the laser pulse to give mesons. It could be possible that some $\mathrm{D}(0)$ cluster type exists inside the metal surface layer, and that the laser pulse can excite them to form mesons even if it is not easily detectable by the $\mathrm{CE}$ experiments: the $\mathrm{CE}$ experiments may use less laser power to dig into the metal surface. It can be observed that the higher Rydberg matter level $\mathrm{D}(1)$ and maybe even $\mathrm{D}(2)$ exist on the surface above the transition temperature for $D(0)$. Thus, it is only the long chain-clusters of $D(0)$ which become unstable above the transition temperature which is expected and agrees with the superfluid properties. However, a fast transformation of $\mathrm{D}(1)$ with $150 \mathrm{pm}$ interatomic distance [5] to $\mathrm{D}(0)$ in $s=1$ with $0.56 \mathrm{pm}$ internuclear distance, required for the meson ejection, seems unlikely. It is apparent that the small $\mathrm{D}(0)$ non-superfluid clusters form another surface phase than the super-fluid long-chain $\mathrm{D}(0)$ clusters. In Fig. 3, the signal of the small clusters at 200-500 ns TOF does not increase with increasing temperature at the transition temperature. This shows that the long-chain clusters do not dissociate to small clusters rapidly but rather decompose to atoms or pairs of atoms which are primarily incorporated in the $\mathrm{D}(1)$ cluster structure which seems to increase at high temperature. Of course, the processes in the surface layer must be understood to be fast processes close to equilibrium, catalyzed by the metal surface. Alternatively, the dissociating longchain clusters could be thought to diffuse into the metal surface; however such a state will still be in close equilibrium with the clusters on the surface. Thus, diffusion into the bulk surface is not believed to be an important or independent process.

The nuclear processes giving the meson formation should also be discussed. Both the kaons and pions observed contain two quarks, while the nucleons in the deuterons each contain three quarks. At present, the best suggestion for the nuclear processes taking place is that two protons with total six quarks form three mesons. This is energetically possible since two protons have a total mass of $2 \times 938 \mathrm{MeV}=1876 \mathrm{MeV}$ and three kaons have approximately $3 \times 495 \mathrm{MeV}=1485 \mathrm{MeV}$ [29-31]. Thus, this process has an excess energy of $361 \mathrm{MeV}$ or $120 \mathrm{MeV}$ per kaon formed, which is quite realistic and in agreement with experimental results. One type of such a process requires the transformation of $\mathrm{d}$ quarks to $\mathrm{s}$ quarks (or $\mathrm{d}$ to $\widehat{\mathrm{s}}$ ), which is possible by the weak interaction [29]. Due to the long interaction time from nanoseconds to days at $<1 \mathrm{pm}$ distance, such processes ought to exist. In this case, mainly charged kaons may be formed $\left(\mathrm{K}^{+}=\right.$ $\mathrm{u}_{\bar{s}}, \mathrm{~K}^{-}=\mathrm{s}_{\bar{u}}$ ) and not so many neutral kaons. This agrees with the present experimental results. Since positive charge dominates in the present experiments, $\mathrm{K}^{+}=\mathrm{u}_{\bar{s}}$ should be formed preferentially from the two protons, possibly as $\mathrm{K}^{+}+\mathrm{K}^{+}+\mathrm{K}_{L}^{0}$.

\section{Conclusions}

The meson-ejecting nuclear processes in $\mathrm{D}(0)$ take place in small $\mathrm{D}(0)$ clusters with typically $3-4$ atoms. These clusters do not form a superfluid phase on the metal target surface and are not directly coupled to the long-chain $\mathrm{D}(0)$ clusters which form a superfluid phase with a Meissner effect as reported previously. The actual $\mathrm{D}(0)$ cluster shape which supports the laser-induced nuclear processes in $\mathrm{D}(0)$ is thus identified.

Acknowledgements This study would not have been possible without the support of Dr. Bernhard Kotzias at Airbus DS, Bremen, Germany. Parts of the equipment used for the experimental studies have been built with the support of GU Ventures $\mathrm{AB}$, the holding company at the University of Gothenburg.

Open Access This article is distributed under the terms of the Creative Commons Attribution 4.0 International License (http://creative commons.org/licenses/by/4.0/), which permits unrestricted use, distribution, and reproduction in any medium, provided you give appropriate credit to the original author(s) and the source, provide a link to the Creative Commons license, and indicate if changes were made.

\section{References}

1. L. Holmlid (2017). PLOS ONE 12, e0169895. https://doi.org/10. 1371/journal.pone.0169895.

2. E. Klempt, C. Batty, and J.-M. Richard (2005). Phys. Rep. 413 , 197.

3. L. Holmlid (2013). Int. J. Mass Spectrom. 352, 1.

4. P. U. Andersson, B. Lönn, and L. Holmlid (2011). Rev. Sci. Instrum. 82, 013503.

5. L. Holmlid (2012). J. Clust. Sci. 23, 95.

6. L. Holmlid (2017). J. Mol. Struct. 1130, 829. https://doi.org/10. 1016/j.molstruc.2016.10.091.

7. D. V. Balin, V. A. Ganzha, S. M. Kozlov, E. M. Maev, G. E. Petrov, M. A. Soroka, G. N. Schapkin, G. G. Semenchuk, V. A. Trofimov, A. A. Vasiliev, A. A. Vorobyov, N. I. Voropaev, C. Petitjean, B. Gartner, B. Lauss, J. Marton, J. Zmeskalc, T. Case, K. M. Crowe, P. Kammel, F. J. Hartmann, and M. P. Faifman (2011). Phys. Part. Nuclei 42, 185.

8. P. U. Andersson, L. Holmlid, and S. R. Fuelling (2012). J. Supercond. Novel Magn. 25, 873.

9. L. Holmlid (2013). Int. J. Mass Spectrom. 351, 61. 
10. S. Badiei, P. U. Andersson, and L. Holmlid (2010). Phys. Scripta 81, 045601.

11. S. Badiei, P. U. Andersson, and L. Holmlid (2010). Appl. Phys. Lett. 96, 124103.

12. L. Holmlid (2011). Int. J. Mass Spectrom. 304, 51

13. L. Holmlid (2018). J. Mol. Struct. 1173, 567. https://doi.org/10. 1016/j.molstruc.2018.06.116.

14. F. Winterberg (2010). J. Fusion Energy 29, 317.

15. F. Winterberg (2010). Phys. Lett. A 374, 2766.

16. T. Guénault Basic Superfluids (Taylor \& Francis, London, 2003).

17. L. Berezhiani, G. Gabadadze, and D. Pirtskhalava (2010). J. High Energy Phys. 2010, 122. https://doi.org/10.1007/JHEP04 (2010)122.

18. P. F. Bedaque, M. I. Buchoff, and A. Cherman (2011). J. High Energy Phys. 2011, 94. https://doi.org/10.1007/JHEP04 (2011)094.

19. E. Babaev, A. Sudbø, and N. W. Ashcroft (2004). Nature 431, 666.

20. L. Holmlid and S. R. Fuelling (2015). J. Cluster Sci. 26, 1153.

21. J. E. Hirsch (2012). Phys. Scr. 85, 035704.
22. P. U. Andersson and L. Holmlid (2011). Phys. Lett. A 375, 1344.

23. L. Holmlid (2015). Int. J. Modern Phys. E 24, 1550026.

24. G. R. Meima and P. G. Menon (2001). Appl. Catal. A 212, 239.

25. M. Muhler, R. Schlögl, and G. Ertl (1992). J. Catal. 138, 413.

26. S. Badiei, P. U. Andersson, and L. Holmlid (2009). Int. J. Mass Spectrom. 282, 70.

27. L. Holmlid (2013). Int. J. Modern Phys. E 22, 1350089.

28. L. Holmlid and B. Kotzias (2016). AIP Adv. 6, 045111.

29. W. E. Burcham and M. Jobes Nuclear and Particle Physics (Pearson, Harlow, 1995).

30. C. Nordling and J. Österman Physics Handbook (Studentlitteratur, Lund, 1988).

31. K. S. Krane Introductory Nuclear Physics (Wiley, Hoboken, 1988).

32. L. Holmlid (2015). Int. J. Modern Phys. E 24, 1550080.

33. L. Holmlid (2016). Int. J. Modern Phys. E 25, 1650085.

34. M. A. Prelas, H. Hora, and G. H. Miley (2014). Phys. Lett. A 378, 2467.

35. L. Holmlid (2013). Laser Part. Beams 31, 715. 